Original Article

\section{Expression of Tp53 and USP7 during development of lungs in mouse model.} Amna Mughal ${ }^{1,2(D)}$, Mushtaq Hussain ${ }^{1}(\mathbb{D})$, Zaheer Amjad ${ }^{1,3}(\mathbb{D})$, Khalida Parveen ${ }^{1,4}$ (D) \& Tazeen Mustansar ${ }^{1,5}$ (D)

${ }^{1}$ Bioinformatics and Molecular Medicine Research Group, Dow Research Institute of Biotechnology and Biomedical Sciences, Dow College of Biotechnology, Dow University of Health Sciences, Karachi-Pakistan

${ }^{2}$ Department of Anatomy, Jinnah Sindh Medical University, Karachi-Pakistan

${ }^{3}$ Department of Anatomy, Dow International Medical College, Dow University of

Health Sciences, Karachi-Pakistan

${ }^{4}$ Department of Anatomy, Shaheed Mohtarma Benazir Bhutto Medical College, Lyari, Karachi-Pakistan

${ }^{5}$ Department of Pathology, Dow Medical College, Dow University of Health Sciences, Karachi-Pakistan
Doi: 10.29052/IJEHSR.v8.i4.2020.173-179

Corresponding Author Email:

mushtaq.hussain@duhs.edu.pk

Received 11/09/2020

Accepted 14/11/2020

First Published 01/12/2020

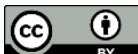

(c) The Author(s). 2020 Open Access This article is distributed under the terms of the Creative Commons Attribution 4.0 International License (http://creativecommons.org/licenses/by/4.0/)

\title{
Abstract
}

Background: Much of the human developmental biology, especially at the molecular resolution, has been investigated on a mouse model. Resultantly, it has been demonstrated that lungs development is underpinned by differential and time course dependent expression of many proteins, including tumor protein 53 (Tp53). However, the expression of Ubiquitin Specific Protease 7 (USP7), a deubiquitinase of Tp53, has not been reported for the process. The study focuses on expressional changes of Tp53 and its stabilizer protein USP7 during the development of lungs.

Methodology: Briefly, mice embryos of three different stages of lungs development, pseudoglandular, canalicular and saccular stages, were retrieved from pregnant mice and subjected to histological studies. Morphometric measurements of tubules diameter, tubular wall thickness and tubular lumen diameter were taken during the three stages. Expressions of Tp53 and USP7 in the developing lungs were investigated using immunohistochemistry and statistically analyzed in terms of optical density.

Results: Anatomically, decreases in tubular diameter and tubule wall thickness were observed from the canalicular to the saccular stage, whereas no significant differences were found between the stages in relation to the luminal diameter of tubules. Expression of Tp53 gradually increased in tubules from the pseudoglandular and saccular stage $(p=0.0169)$. However, no difference was observed in interstitial tissue in all three stages. In comparison, USP7 expression was found to increase from pseudoglandular to the canalicular stage in both tubules and interstitial tissue. A positive correlation was only observed between Tp53 and USP7 expression in the interstitial tissue.

Conclusion: The results suggest expression of Tp53 is mainly regulated by USP7 during lungs development in tubules and interstitial tissue, especially between canalicular and saccular stages of lungs development.

\section{Keywords}

Lungs Development, Tumor Protein 53, Ubiquitin Specific Protease 7, Immunohistochemistry.

\section{Check for updates}




\section{Introduction}

Mice are amongst the most profoundly model organism to study human development ${ }^{1}$. Over $95 \%$ of the human genes have direct orthologues being present in mice. Consistently, both species' developmental process also holds considerable similarities both at the gross and molecular resolutions ${ }^{2}$.

In mice, organogenesis of lungs also resembles with humans and divided into five stages namely, embryonic (Embryonic day (E) 9.5 to the E12.5), pseudo glandular (E12.5 to E16.5), canalicular (E16.5 to E17.5), saccular (E17.5 to Postnatal Day (PD) 5) and alveolar (PD5 to PD30) stages. These days are alignable to lungs development in human from 4th week to 7th week of gestation (embryonic stage), 7th to 16th week (pseudoglandular stage), 17th to 26th week (canalicular stage), 26th to 36th week (saccular stage) and 36th to 2 years (alveolar stage) ${ }^{3}$. To date, regulation and expressional changes in several proteins are linked with lungs development. Amongst those most notable are: Nkx2.1, BMP4, FGF10, FGF7, SHH, FOXA2, PDGFRA, SOX9 and FBXO11 ${ }^{4-6}$. Of these, Tp53 holds profound importance ${ }^{7}$.

Tp53, a 393 amino acid long protein, is mainly a proapoptotic protein and considered one of the major proteins involved in the cell cycle regulation ${ }^{8}$. Previously the focus on Tp53 was related to cancer and tumor suppression. However, with recent advances, Tp53 is now evolved as a gene also involved in cell differentiation and developmental processes ${ }^{9}$. An increasing body of information now being available suggests that Tp53 also contributes to developing several organs like the brain, heart, kidney and liver ${ }^{10-13}$. Overexpression of Tp53 has been noticed within proliferating and differentiating alveolar epithelial cells role during lungs developmental process in pseudoglandular and canalicular stages in mice ${ }^{7}$. Although Tp53 is amongst the major transcriptional factor $^{14}$, its expression is regulated by different mechanisms, including the interplay of polyubiquitination and deubiquitination ${ }^{15}$. Ubiquitination of Tp53 is mediated by two different E3 ligases, MDM2 and MDM4, which attach ubiquitin to Tp53, causing its destabilization by making it susceptible to the proteosomal degradation ${ }^{16}$. The process is reversed by deubiquitination mediated by deubiquitinases. Amongst those, USP7 is considered as a key molecule that stabilizes the Tp53 by removing the tagged ubiquitin from the protein rendering its stabilization ${ }^{17}$. USP7, also known as HAUSP (Herpes Virus Associated USP), one of the well-known deubiquitinase, pleotropically regulates the turnover of many cellular proteins, including Tp53, which are collectively involved in tumor suppression, DNA damage response, immune response, DNA replication and transcription ${ }^{18}$. In addition, the role of USP7 has also been demonstrated in developmental processes. Deletion of USP7 in mice resulted in embryonic lethality from E6.5 and E7.5 $5^{19}$. Moreover, USP7 deletion in mice embryo's neuronal cells showed hypoplasia and other abnormalities of brain development, suggesting its role in brain organogenesis ${ }^{20}$.

As mentioned above, Tp53 has been reported to be involved in the lungs' developmental processes; however, the expression of its regulator, USP7, has not been investigated in a similar context. Herein we present the alignment of USP7 expression with its partner protein Tp53 during the three main lungs developmental stages in mice.

\section{Methodology}

Sample Collection A total of 7 pregnant mice of NMRI strain were taken in the study ranging in weight from 32 to $46 \mathrm{gm}$ and corresponding to the gestational age of E14 to E20 days (Figure $1 \mathrm{~A}-\mathrm{C}$ ). Embryos were retrieved from the mice and judged for the age in terms of pseudoglandular, canalicular and saccular stages of lungs development on the basis of their body weight and characteristic features of the fetuses. The Institutional Board Review approved the study and all experimental protocols for Animal Research and Ethics, Dow University of Health Sciences, Karachi, Pakistan.

Sample Processing Embryos belonging to pseudoglandular, canalicular, and saccular lungs development stages were separated and fixed in $10 \%$ neutral buffer formalin. Sagittal and coronal sections of embryos were taken, followed by embedding in paraffin blocks. Multiple $4 \mu \mathrm{m}$ thick sections of paraffinembedded blocks were subjected to Hematoxylin and Eosin (H\&E) staining and immunostaining for Tp53 and USP7. 
Immunohistochemistry To observe the expression of Tp53 and USP7, sections were first deparaffinized and rehydrated. Antigen retrieval was done using sodiumcitrate buffer $(\mathrm{pH}$ 6.0) followed by incubation in $3 \%$ hydrogen peroxide for $10 \mathrm{~min}$. Endogenous peroxidase activity was blocked using a blocking solution (animal serum with concentration: $\leq 10 \mathrm{EU} / \mathrm{mg}$ ) for $10 \mathrm{~min}$ at room temperature. The sections were then probed with primary antibodies: anti-mouse USP7 antibody (NB100513, 1:4000), anti-mouse Tp53 antibody (NB200-103, 1:400) followed by secondary antibodies: HRP conjugated secondary antibodies (NB7160, PHJ170515). DAB substrate system (DAB-060) was used with counterstaining with $\mathrm{H} \& \mathrm{E}$ to view the expression of Tp53 and USP7 on the sections.

Imaging \& quantification of expression Images of the sections were taken using the Optika camera microscope. The H\&E and immune stained images were analyzed using ImageJ software. Immunoexpression of the Tp53 and USP7 was monitored using the $\mathrm{IHC}$ profiler plugin of ImageJ software under three different thresholds of high positive, positive and low positive and values were later coalesced as optical density according to the formulation developed by Seyed Jafari SM ${ }^{21}$.

Statistical analysis Data were analyzed using GraphPad prism version V5. The distribution of data was assessed using the Kolmogorov-Smirnov test. The difference among the variable was analyzed using Mann Whitney t-test. The correlation of the expression of Tp53 and USP7 was performed using linear regression graphs with 95\% confidence, and a p-value of $\leq 0.05$ was considered significant in all cases.

\section{Results}

Pseudoglandular stage of lungs development was characterized by columnar epithelial lined tubules surrounded by a layer of smooth muscle fibers and the newly formed blood vessels within the interstitial tissue. During the lungs' canalicular stage, tubules lined by cuboidal epithelium, vessels, and abundant interstitial tissue were observed evident. Changes found in the saccular stages were noticed as the presence of numerous small sacs lined by squamous epithelium and a decrease in the amount of interstitial tissue in between the sacs (Figure 1A-F)

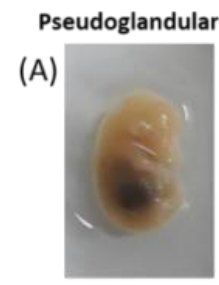

(D)

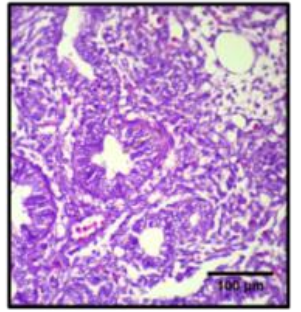

(G)

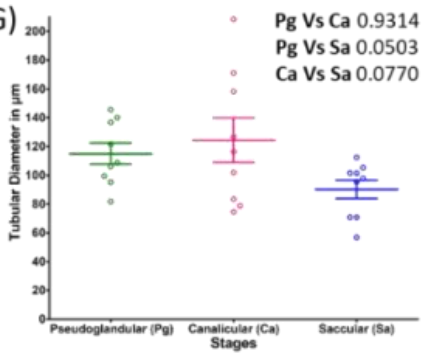

(B)

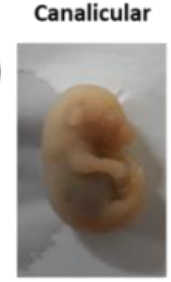

(E)

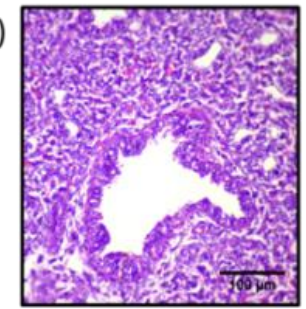

(H)

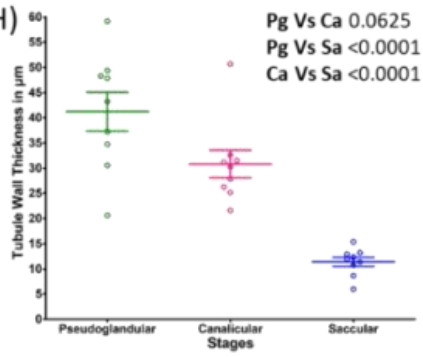

(C) Saccular
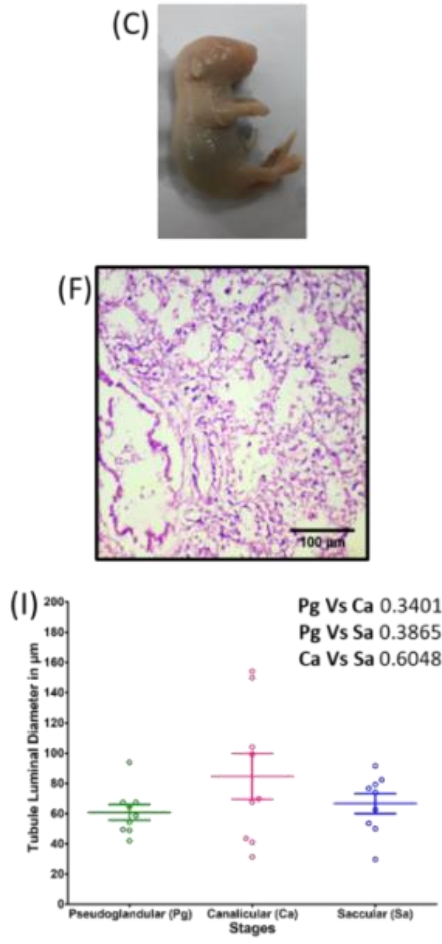

Figure 1: Micrometric analysis of developing lungs in mice. Photographs of mice fetuses of different stages pseudoglandular (A), canalicular (B) and saccular (C) are shown with representative histomicrographs, respectively (D-F). Graphs showing measurements 
of $(\mathrm{G})$ tubular diameter, $(\mathrm{H})$ tubular wall thickness, and $(\mathrm{I})$ lumen diameter, where large and small horizontal bars represent mean and standard deviations, respectively. Values of statistical significance are shown within the graphs.

Micrometric analyses of tubular diameter showed no statistically significant difference from the pseudoglandular to the canalicular stage, whereas a statistically significant difference $(p=0.0770)$ was observed and a decrease was noticed from canalicular to the saccular stage (Figure 1D-G). Tubular wall thickness decreased as the developmental stage transitions from pseudoglandular to canalicular and then to saccular stages. However, a significant difference was only observed between canalicular and saccular stages $(p<0.0001) \quad$ (Figure 1D-F; H). In comparison, the lumen of tubules remained unchanged during development from pseudoglandular to saccular stages (Figure 1D-F; I).

Expression of Tp53 was found to gradually increased from pseudoglandular to canalicular stages and then to saccular stages. However, a statistically significant difference ( $p=0.0169)$ was only observed between the starting and endpoint of assessment, namely, pseudoglandular and saccular stages, respectively. Conversely, no difference in the expression of the Tp53 was observed in interstitial tissues (Figure 2A-E). Interestingly, like Tp53, expression of USP7 was found unchanged in interstitial tissues between canalicular and saccular stages. However, found increased when the expression of the protein was assessed between pseudoglandular and canalicular stages in both tubules $(p=0.0108)$ and interstitial tissues $(p=0.0082)$ (Figure $2 F-$ J).

\section{USP7 Expression}

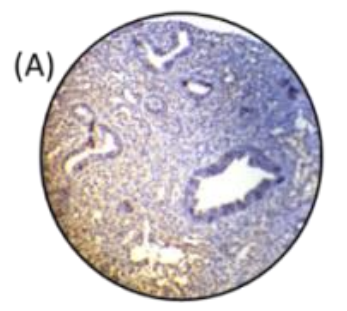

(D)
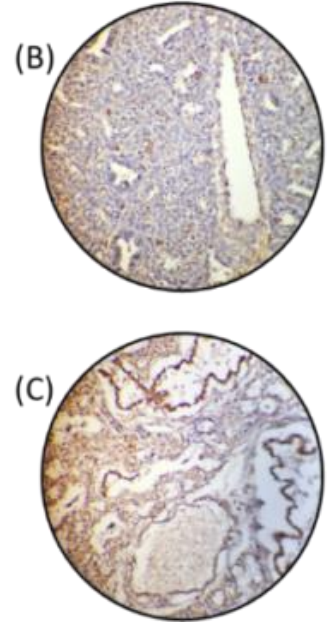

(E)
Tubular Expression
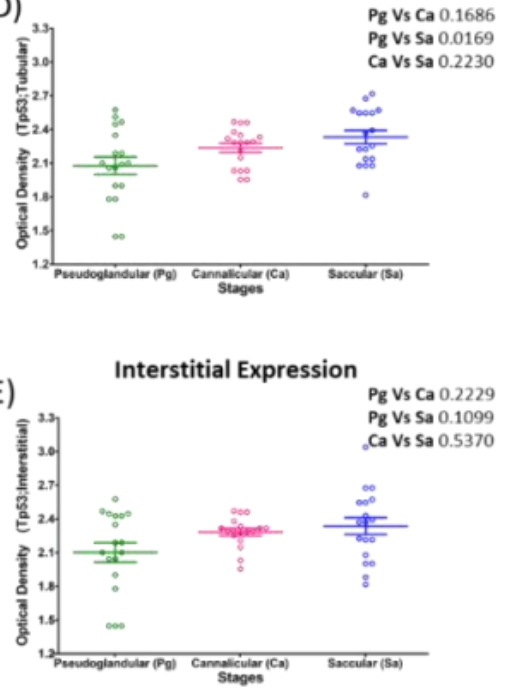

(F)
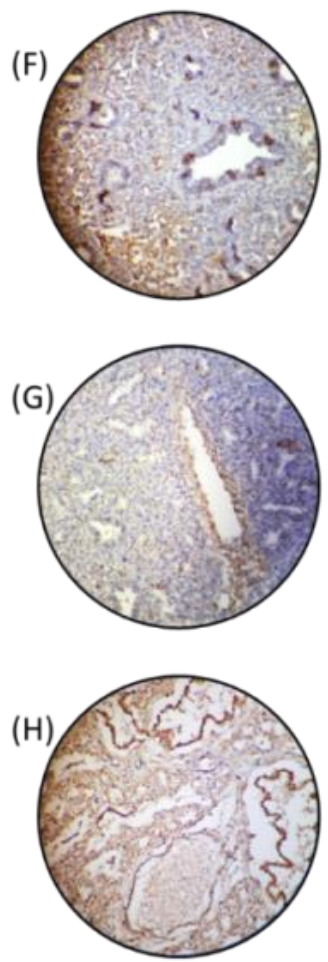

(I)

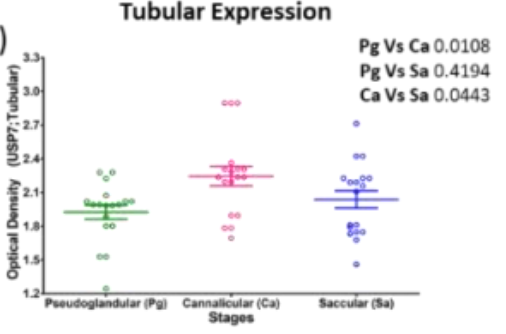

(J)

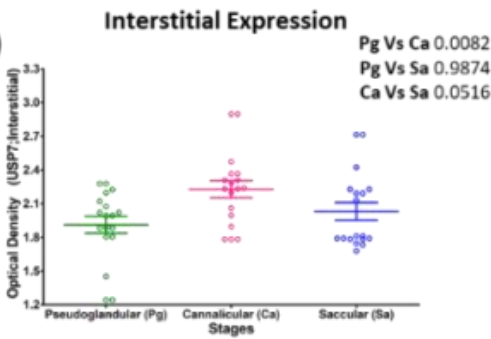

Figure 2: Expression of Tp53 and USP7 in developing lungs of mice. Representative histomicrographs of Tp53 expression in three different stages of lungs development, pseudoglandular (A), canalicular (B) and saccular (C). The difference in the Tp53 expression in terms of optical density between the developing lungs stages are also shown in tubules (D) and interstitial tissues (E). Representative histomicrographs of USP7 expression in three different stages of lungs development, pseudoglandular (F), canalicular $(\mathrm{G})$ and saccular $(\mathrm{H})$. The difference in the USP7 expression in terms of optical density between the developing lungs stages are also shown in tubules (I) and interstitial tissues (J). 
This later dwindles to the level of pseudoglandular stage when the developmental phase transited from canalicular stage to saccular stage in both cases. Importantly, correlation analyses showed a positive correlation between Tp53 and USP7 in the case of only interstitial tissues (Figure 3).

(A)

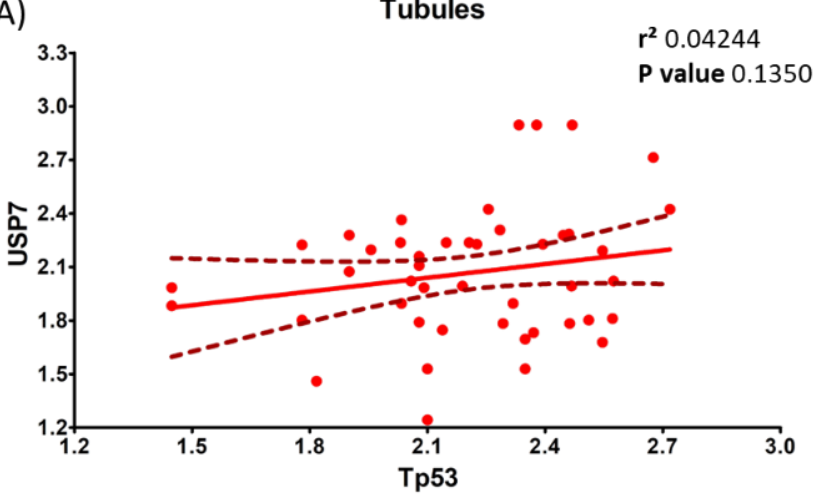

(B)

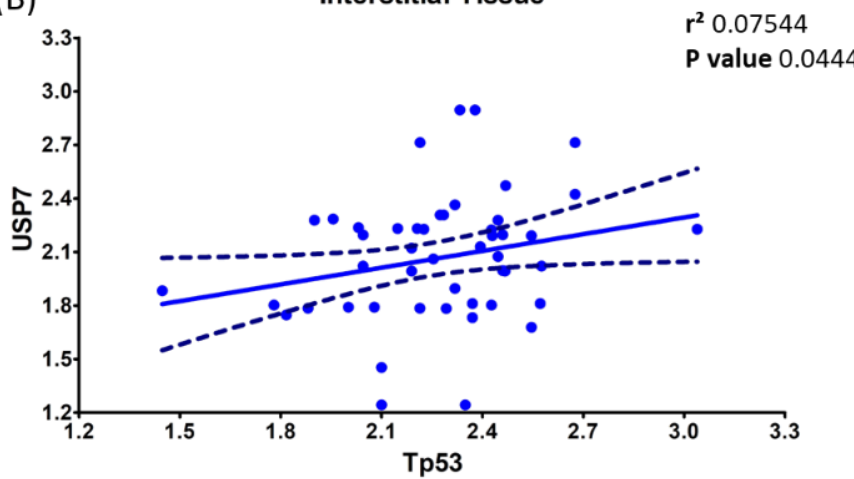

Figure 3: Correlation between Tp53 and USP7 expression. Graphs showing the correlation between expression values (optical density) of Tp53 and USP7 in (A) tubules and (B) interstitial tissues. The solid and broken lines represent trend lines and 95\% confidence interval bands, respectively.

\section{Discussion}

Development of lungs in mice starts with the appearance of bud from the ventral foregut on E9.5 and continues to the $\mathrm{PD}^{4}{ }^{4}$. On the basis of morphological changes of the developing airways, lungs development is divided into five stages ${ }^{22}$. Of these five stages of lungs development, three stages, namely pseudoglandular, canalicular and saccular stages, are included in our study as they encompass major events of lungs organogenesis. These changes are comparable to the lungs development in humans from 7 th week 36 weeks of gestation ${ }^{4}$.

In the pseudoglandular stage, the airway tubule undergoes branching morphogenesis and is embedded in the surrounding mesenchymal tissue, which begins to differentiate into cartilage, smooth muscle fibers and blood vasculature. In the canalicular stage, branching continues with a concomitant increase in size and number of airway tubules, decreasing the surrounding interstitial tissue and capillaries coming in close proximity to tubules ${ }^{23}$. In the saccular stage, airspaces appear as thin epithelial lined saccules with a gradual increase in size and decrease in surrounding interstitial tissue ${ }^{3}$.

Histological changes as observed during the lungs development in the mice in the present study such as the presence of transition from columnar to the cuboidal epithelium, layer of smooth muscle fibers surrounding tubules, formation of blood vessels within interstitial tissue, and formation of numerous sac lined by squamous epithelium are consistent with the anatomical changes previously reported in connection to the lungs development in mice ${ }^{24-26}$. Although no change in the tubular lumen was observed during the lungs' developmental stages while both tubular diameter and tubular wall thickness tend to decrease with advancing stages of lungs development. This observation is in line with the earlier studies ${ }^{27}$. The decrease in the wall thickness and tubular diameter may, in turn, facilitate the accommodation of an increasing number of tubules during the lungs development within a space. The increasing number of tubules is an essential feature to develop fully functional lungs in relation to the transient oxygen reservoir during breathing.

The role of USP7 in mice's development process has been explored by Kon et al., who developed USP7 knockout mice. These knockout mice embryos died in their early embryonic period from E6.5 and E7. $5^{19}$. In another experiment, deletion of USP7 in the neuronal cell of mice embryo resulted in abnormal brain development like flat forebrain, absence of cerebellum and hypoplasia ${ }^{20}$. The developing stage, E15.5, have 
shown elevated expression of Tp53 in the developing lungs. Additionally, Tp53 deficient mice embryos exhibited developmental abnormalities like exencephaly, cleft palate and abnormal lungs architecture $^{6}$. Overexpression of Tp53 in the developing alveolar epithelial cells suggests Tp53 role in alveolar type I and alveolar type II cell differentiation, which is the key factor in the formation of gas exchange units $^{7}$. Accounting for this, it is conceivable that both Tp53 and its stabilizer USP7 works concurrently in lungs development. Elevated expression of USP7 during the canalicular stage and later elevation of Tp53 in the saccular stage is in line with the molecular functionality of USP7. Since USP7 is amongst the major deubiquitinating enzyme for $T p 53^{17}$, higher expression of USP7 (in canalicular stage) preceded the elevated expression of Tp53 (in saccular stage), suggesting the generation of the increased repertoire of USP7 during the canalicular stage. Consequently, this later leads to the stabilization of Tp53 in the saccular stage. Tp53 is a well-known cell cycle regulator due to its role in DNA repair and checkpoints of the cell cycle ${ }^{28}$. Thereby, constant expression of Tp53 during the three stages of lungs development is very likely to be the indicator of a rapid increase in the cellular density in the lungs. Consistently, to maintain the desired turnover of Tp53 in the cells, a stabilizer, namely USP7, is required ${ }^{17}$. Therefore, upregulation of USP7 from pseudoglandular to the canalicular stage is potentially representing a molecular event that set the environment for constant expression of Tp53 to carry out the destined function in the subsequent stages of lungs development. However, it is worth mentioning here that augmenting the present study with assessment of expression of Tp53 ubiquitin ligases like MDM2 and MDM4 and its other relatively less common stabilizers like CYLD could shed more light on the molecular events that underpin lungs development.

\section{Conclusion}

The present investigation points to the role of USP7 in relation to the stabilization of Tp53 during lungs development, which is previously not reported. However, more studies are warranted for other Tp53 partner proteins to gain detailed molecular resolution of its working during lungs development.

\section{Conflicts of Interest}

The authors declare no competing interest.

\section{Acknowledgement}

The authors would like to acknowledge the animal house of Dow University of Health Sciences for the provision of animals.

\section{Funding}

None

\section{References}

1. Perlman RL. Mouse models of human disease: An evolutionary perspective. Evol Med Public Health, 2016; (1):170-176.

2. Cunha G, Overland $M$, Li $Y$, Cao $M$, Shen J, Sinclair A, Baskin L. Methods for studying human organogenesis. Differentiation. 2016; 91(4-5):10-14.

3. Schittny JC. Development of the lungs. Cell Tissue Res. 2017; 367(3):427-444.

4. Rankin SA, Zorn AM. Gene regulatory networks governing lungs specification. J Cell Biochem. 2014; 115(8):1343-1350.

5. Chen YW, Huang SX, De Carvalho AL, Ho SH, Islam MN, Volpi S, Notarangelo LD, Ciancanelli M, Casanova JL, Bhattacharya J, Liang AF. A three-dimensional model of human lungs development and disease from pluripotent stem cells. Nat Cell Biol. 2017; 19(5):542-549.

6. Tateossian H, Morse S, Simon MM, Dean CH, Brown SD. Interactions between the otitis media gene, Fbxo11, and p53 in the mouse embryonic lungs. Dis Model Mech. 2015; 8(12): 1531-1542.

7. Tebar M, Boex JJ, Ten Have - Opbroek AA. Functional overexpression of wild-type p53 correlates with alveolar cell differentiation in the developing human lungs. Anat. Rec. 2001; 263(1): 25-34.

8. Ter Huurne M, Peng $T, Y i$, van Mierlo G, Marks $H$, Stunnenberg $\mathrm{HG}$. Critical role for P53 in regulating the cell cycle of ground state embryonic stem cells. Stem Cell Rep. 2020; 11;14(2):175-83.

9. Jain AK, Barton MC. p53: emerging roles in stem cells, development and beyond. Development. 2018; 15; 145(8):dev158360.

10. Mendrysa SM, Ghassemifar S, Malek R. p53 in the CNS: Perspectives on development, stem cells, and cancer. Genes Cancer. 2011; 2(4):431-442.

11. Zhang Q, He X, Chen L, Zhang C, Gao X, Yang Z, Liu G. Synergistic regulation of p53 by $\mathrm{Mdm} 2$ and $\mathrm{Mdm} 4$ is critical in cardiac endocardial cushion morphogenesis during heart development. J Pathol. 2012; 228(3):416-428. 
12. Saifudeen Z, Dipp S, Stefkova J, Yao X, Lookabaugh S, ElDahr SS. p53 regulates metanephric development. J Am Soc Nephrol. 2009; 20(11):2328-2337.

13. Chen LJ, Hsu CC, Hong JR, Jou LK, Tseng HC, Wu JL, Liou YC, Her GM. Liver- specific expression of p53-negative regulator $\mathrm{mdm} 2$ leads to growth retardation and fragile liver in zebrafish. Dev Dyn. 2008; 237(4):1070-1081.

14. Sullivan KD, Galbraith MD, Andrysik Z, Espinosa JM. Mechanisms of transcriptional regulation by p53. Cell Death Differ. 2018; 25(1):133-143.

15. Kwon SK, Saindane M, Baek KH. p53 stability is regulated by diverse deubiquitinating enzymes. Biochim Biophys Acta Rev Cancer. 2017; 1; 1868(2):404-411.

16. Tan BX, Liew HP, Chua JS, Ghadessy FJ, Tan YS, Lane DP, Coffill CR. Anatomy of Mdm2 and Mdm4 in evolution. J. Mol. Cell Biol. 2017; 1;9(1):3-15.

17. Li M, Chen D, Shiloh A, Luo J, Nikolaev AY, Qin J, Gu W. Deubiquitination of p53 by HAUSP is an important pathway for p53 stabilization. Nature. 2002; 416(6881): 648-653.

18. Georges A, Marcon E, Greenblatt J, Frappier L. Identification and characterization of USP7 targets in cancer cells. Sci Rep. 2018; 26;8(1):1-2.

19. Kon N, Kobayashi Y, Li M, Brooks CL, Ludwig T, Gu W. Inactivation of HAUSP in vivo modulates p53 function. Oncogene. 2010; 29(9):1270-1279.
20. Kon N, Zhong J, Kobayashi Y, Li M, Szabolcs M, Ludwig T, Canoll PD, Gu W. Roles of HAUSP-mediated p53 regulation in central nervous system development. Cell Death Differ. 2011; 18(8):1366-1375.

21. Seyed Jafari SM, Hunger R. IHC Optical Density Score: A New Practical Method for Quantitative Immunohistochemistry Image Analysis. Appl. Immunohistochem. Mol Morphol. 2017; 25(1):e12-3.

22. Carlson BM. Human Embryology and Developmental biology E-book. 2018: Elsevier Health Sciences.

23. Nikolić MZ, Sun D, Rawlins EL. Human lungs development: Recent progress and new challenges. Development. 2018; 15; 145: dev163485

24. Copland I, Post M. Lungs development and fetal lungs growth. Paediatr Respir Rev. 2004; 5: S259-S264.

25. Joshi S, Kotecha S. Lungs growth and development. Early Hum Dev. 2007; 83(12): 789-794.

26. Herriges $M$, Morrisey EE. Lungs development: orchestrating the generation and regeneration of a complex organ. Development. 2014; 141(3): 502-513.

27. Jeffery PK. The development of large and small airways. Am J Respir Crit. 1998; 157(5): S174-S180.

28. Shaw $\mathrm{PH}$. The role of $\mathrm{p} 53$ in cell cycle regulation. Pathol Res Pract. 1996;192(7):669-675. 